\title{
Can We Predict Future Depression among Residents before the Start of Clinical
}

\section{Training?}

Makoto Ito, ${ }^{1}$ Emiko Seo, ${ }^{1,2}$ Ryoko Ogawa, ${ }^{2}$ Masaru Sanuki, ${ }^{1,2}$ Takami Maeno, ${ }^{1}$ \& Tetsuhiro Maeno ${ }^{1,2}$

${ }^{1}$ Graduate School of Comprehensive Human Sciences, University of Tsukuba, Tsukuba, Ibaraki, Japan

${ }^{2}$ Center for Medical Education and Training, University of Tsukuba Hospital, Tsukuba, Ibaraki, Japan

\begin{abstract}
CONTEXT: Depression among medical residents is a very serious problem. It is, however, very difficult to detect signs of depression early, despite the severity and frequency of depression. Therefore we designed a nationwide longitudinal study to investigate whether the sense of coherence (SOC) scale, an indicator of stress-coping ability, could predict future depressive symptoms among medical residents.
\end{abstract}

METHODS: We distributed self-administered questionnaires to first-year residents in 251 postgraduate educational hospitals just before the start of their clinical training. The questionnaire contained the Center for Epidemiologic Studies Depression (CES-D) scale (a screening tool for depression), the SOC scale, and demographic factors. After three months, we again distributed questionnaires to the residents who responded to the first survey. The second questionnaire contained the CES-D scale, and their working conditions. We categorised the respondents into three groups according to their SOC score, and we analysed the relationship between the three SOC groups (low, middle, high) 
and depressive symptoms at the follow-up survey.

RESULTS: In all, 1738 of 2935 residents (59.2\%) responded to the first survey. Of these, 1245 residents (71.6\%) also responded to the follow-up survey. A total of 189 residents were excluded because they screened positive for depressive symptoms at the first survey. At the follow-up survey, 238 residents (23.3\%) had new-onset depressive symptoms, with $41.2 \%$ in the low SOC group, $22.3 \%$ in the middle group, and $11.3 \%$ in the high group ( $\mathrm{p}<0.01$ ). The odds ratio of the low SOC group for new-onset depressive symptoms, adjusted for demographic factors, baseline CES-D score and mean working time, was 3.11 (95\% confidence interval, 1.48-6.53), with the high SOC group as the reference.

CONCLUSIONS: The SOC score was significantly related to future depressive symptoms among medical residents. The SOC scale might be a useful and easy-to-use predictor of future depression. 


\section{INTRODUCTION}

The medical residency period is important for creating skilful doctors; however it is stressful and burdensome, not only physically, but mentally ${ }^{1}$. Residents face very high levels of stress; ${ }^{2}$ furthermore, depression is one of the most important reactions to stress, ${ }^{3}$ and may lead to suicide which is an especially significant problem. As well as the stresses facing general physicians or consultants, such as patient-related factors and heavy workloads, the residents also face particular stresses, such as adjusting to relocation, inadequate coping skills, and the leap from being just a student to being a responsible resident. ${ }^{4-6}$ Goebert et al. ${ }^{7}$ reported suicidal ideation was present in nearly 6\% of depressed residents. The suicide rate among male physicians is $40 \%$ higher than in the general male population and that of female physicians is $130 \%$ higher than in the general female population. ${ }^{8}$

Depression among residents is also harmful to patients. Fahrenkopf et al. ${ }^{9}$ reported that depressed residents made 6.2 times as many medical errors per month as residents who were not depressed. Thus depression of residents is related to medical error and medical error may lead to depression, ${ }^{9}$ making doctors 'the second victim'. ${ }^{10}$

The prevalence of depression among residents is variable, with reports ranging from $7 \%-35 \%{ }^{11-14}$ however most studies have found the rate of depression among residents to be higher than that of the general public (4\%-5\%). ${ }^{11,14,15}$ In fact, we have previously reported that $25.5 \%$ of first year residents, who had been without depressive symptoms before the start of training, had depressive symptoms two months after the start of clinical training. ${ }^{16}$

General preventive interventions for depression among residents include restriction of working time ${ }^{17}$, mental health services ${ }^{3}$, and sharing the information of residents' 
mental health among directors and attending physicians. ${ }^{18}$ But using only these general interventions still makes it difficult to prevent depression among residents.

The early detection of depression and the provision of appropriate care are essential for creating a healthy clinical training environment; however, early detection of depression is difficult, despite the severity and frequency of the condition. ${ }^{8}$ This may be due to the poor recognition of depression among both resident colleagues and attending physicians. ${ }^{8}$ In addition, many residents do not know exactly how to get help for mental health problems or worry about disclosing the fact to colleagues or attending physicians. ${ }^{6}$ If a predictor for the group at high risk of depression can be identified, the program director and attending physician could monitor this high risk group. This may help the early detection of depression in residents and facilitate the provision of appropriate support before depression gets any worse. ${ }^{6}$

Previous studies have suggested some predictive factors for depressive symptoms in residents, such as self-criticism ${ }^{19}$, an unhappy childhood ${ }^{2}$, and previous mental health problems. ${ }^{14}$ However these factors are intimate and sensitive and many residents may hesitate to answer questions about such personal issues, so, in practice, it might be hard to use predictive factors such as these to detect the high risk group for depression. A previous study has also suggested the sense of coherence (SOC) score has an association with the onset of depression among Japanese workers, where the risk ratio of a high SOC compared to a low SOC for depression was $0.18 .{ }^{20}$ A SOC was one of the concepts stated by Antonovsky in 1979, indicating a stress-coping ability. Since then, the SOC scale has been used as a tool to measure stress-coping ability, and a number of previous studies have reported that people with high SOC scores can maintain good mental health. ${ }^{21}$ Haoka et al. ${ }^{22}$ also suggested a low SOC score in a group of residents indicated poorer 
mental health, compared to a group of residents with a high SOC score, and it could influence the way job stress was perceived. That cross sectional study, however, could not demonstrate that the SOC score might predict future depression among residents.

So in the present longitudinal study, we investigated the possibility that the SOC score, when evaluated before the start of clinical training, is a predictor of future depressive symptoms among medical residents. 


\section{METHODS}

\section{Subjects and study design}

We contacted 853 postgraduate educational hospitals across Japan using REIS (Residency Electronic Information System), which is the Japanese government database of hospitals providing clinical training. We asked them to participate in our study, and then mailed questionnaires to the hospitals which agreed to participate, and the clinical training program directors distributed the questionnaires to first-year residents, at the orientation session at the beginning of clinical training. The questionnaire contained the Center for Epidemiologic Studies Depression (CES-D) scale as an evaluating tool for depressive symptoms, the SOC scale and basic demographic information, such as age, sex and type of hospital (university hospital or other educational hospital). In addition, we allocated an ID number to each questionnaire, which was also used for the follow-up survey. The ID number was handled separately to protect personal information.

Three months after the start of their training, we conducted a follow-up survey of only those residents who had responded to the first survey. The follow-up questionnaire contained the CES-D scale, and questions about working conditions, such as mean working time on weekdays, mean working time on weekends, and monthly number of night duties. This series of surveys was conducted in 2011.

\section{CES-D scale}

We used the CES-D scale to evaluate depressive symptoms. The CES-D scale was developed as a case-finding tool to screen for depressive symptoms in the general population by the National Institute of Mental Health, and it is widely used as a self-report scale to measure symptoms associated with depression. ${ }^{23}$ The CES-D tool 
consists of 20 items that focus on depressive symptoms within the last week, and, on a scale from zero to three, respondents rate all 20 items, scoring from 0 to $60 .{ }^{23}$ We used the Japanese version of the CES-D scale; the reliability and validity of this version have been confirmed, and using 16 points as a cut-off, its sensitivity is $88.2 \%$ and specificity is 84.8\%. ${ }^{24}$ A person with a score of more than 16 was defined as screening positive for depressive symptoms. ${ }^{24}$

\section{SOC scale}

We used the Japanese version of the SOC questionnaire consisting of 13 items rated on 5-point scales, which has been previously tested for reliability and validity. ${ }^{25}$ We classified residents into three groups based on their SOC score. Because there is no definitive cut-off point for the SOC scale, we used the mean and standard deviation (SD) of the total SOC score to define the groups, as in previous studies. ${ }^{22,26}$ Groups were as follows: low SOC score group, SOC score < mean - SD; high SOC score group, SOC score $>$ mean $+\mathrm{SD}$; middle SOC score group, SOC score $\geq$ mean $-\mathrm{SD}$ but $\leq$ mean $+\mathrm{SD}$.

\section{Mean working time}

As in previous studies, ${ }^{22,27}$ we thought it might be difficult for residents to directly state their mean working time per week as most of them recognise their working time by the hours per day, so their mean weekly working time was calculated with the following equation using three components - mean working time on weekdays, mean working time on weekends, and monthly number of night duties: Mean weekly working time $=$ [5 x (mean working time on weekdays) $]+[2 \mathrm{x}$ (mean working time on weekends) $]+$ [7 x (monthly number of night duties/30) x (24 - mean working time on weekdays)]. 
That is, the mean weekly working time was defined as the sum of five times the mean daily working time on weekdays, plus two times the mean daily working time on weekends, plus the mean working time of night duties. The questionnaire included 'monthly' number of night duties, thus we re-calculated this value as the 'weekly' number of night duties. We calculated the working time of each night duty as ' 24 hours minus the mean daily working time on weekdays'.

\section{Statistical strategy}

We included the residents who answered both the first survey and follow-up survey. We excluded residents with missing data for SOC score, CES-D score, or demographic information. To evaluate how medical training influences their mental health as a stress factor, we also excluded the residents who had already screened positive for depressive symptoms at the first survey, before starting clinical training. Residents who did not screen positive for depressive symptoms at the first survey but screened positive for depressive symptoms at the follow-up survey were defined as residents with 'new-onset depressive symptoms’.

To reveal any associations between new-onset depressive symptoms and the three SOC groups, baseline CES-D score, demographic factors, and working hours, we conducted chi-square tests or $t$ tests for each variable, as univariate analyses. We also calculated the correlation between baseline SOC and CES-D scores. We conducted binomial logistic analysis for new-onset depressive symptoms to adjust for confounding factors, using demographic information and variables with p-values $<0.10$ at the univariate analysis as independent variables. The significance level was set at $\mathrm{p}<0.05$. All statistical analyses were conducted by SPSS version 22.0. 


\section{Ethical consideration}

The study was approved by the Ethics Committee of the Faculty of Medicine of the University of Tsukuba. The purpose of this study and provision of secure data management was stated on the first page of the questionnaire. At the same time we explained to them that their participation in the study was purely voluntary. Results were analysed separately from personal information, to allow for anonymity and confidentiality of personal information. 


\section{RESULTS}

\section{Subject characteristics}

In all, 251 hospitals participated in our study, and we distributed questionnaires to 2935 residents. For the first survey, 1738 residents (59.2\%) responded. Of these, 1245 residents (71.6\%) responded to the follow-up survey. A total of 36 questionnaires had missing data so these respondents were excluded. Of the remaining 1209 residents, 189 residents (15.6\%) screened positive for depressive symptoms at the time of the baseline survey, so they were also excluded from the study, leaving a final number of 1020 residents (82.0\%) subject to analysis in this study. Of these, the number of male residents was 668 (65.5\%). Their mean working time was $78.8 \pm 13.6$ hours per week and the percentage of residents working more than 80 hours per week was $45.7 \%$. The demographic factors, mean SOC score and category, and mean working time of the residents are shown in Table 1.

\section{SOC scores and grouping}

The mean SOC score $( \pm \mathrm{SD})$ of residents was $44.4 \pm 6.0$; the total SOC score ranged from 13 to 65. The correlation between SOC score and CES-D score at baseline was -0.488 (p $<0.01)$.

In all, 148 residents (14.5\%) were categorised in the low SOC score group, 712 residents (69.8\%) in the middle SOC score group, and 160 residents $(15.7 \%)$ in the high SOC score group. Age was the only demographic factor significantly different between the three SOC score groups, with older residents having a higher SOC score than the younger residents. 


\section{New-onset depressive symptoms}

At the follow-up survey, the number of residents with new-onset depressive symptoms was 238 (23.3\%). The demographic factors, mean SOC score and category, and mean working time of the residents with and without new-onset depressive symptoms are shown in Table 2.

In a univariate analysis investigating the association of new-onset depressive symptoms with demographic factors, baseline CES-D score and working time, only the CES-D score at baseline (6.28 \pm 3.8 vs. $8.95 \pm 3.7$, for absence vs. presence of new-onset depressive symptoms, respectively; $\mathrm{p}<0.01$ ) and the mean number of working hours per week (77.5 \pm 13.5 vs. $83.1 \pm 13.9$, for absence vs. presence of new-onset depressive symptoms, respectively; $\mathrm{p}<0.01$ ) were significant. There were no significant differences between sexes (Table 2).

\section{Relationship between new-onset depressive symptoms and SOC score}

The mean SOC score was significantly lower in the residents with new-onset depressive symptoms at follow-up, compared to those residents without new-onset depressive symptoms (Table $2, \mathrm{p}<0.01$ ). The percentage of residents with new-onset depressive symptoms in each SOC score group was as follows: low, 41.2\%; middle, 22.3\%; and high, $11.3 \%(\mathrm{p}<0.01$; Figure 1).

\section{Logistic regression analysis}

The results of multivariate logistic regression analysis for new-onset depressive symptoms are shown in Table 3. The adjusted odds ratio of the low SOC score group for new-onset depressive symptoms was 3.11 (95\% confidence interval [CI], 1.48-6.53; p < 
0.01); the reference was the high SOC score group.

In addition, when we used the SOC score as a continuous variable in a logistic regression analysis, we confirmed that the SOC score was independently related to new-onset depressive symptoms, after adjustment for the baseline CES-D score (odds ratio, 0.93; 95\% CI, 0.90-0.96; $\mathrm{p}<0.01)$. 


\section{DISCUSSION}

In the present longitudinal study, the SOC score had a significant independent association with new-onset depressive symptoms, even after adjustment for the CES-D score at baseline, with the adjusted odds ratio of the low SOC group being 3.11 (the reference was the high SOC group). This suggests that the residents categorized in the low SOC group had a threefold higher risk of future depressive symptoms than those in the high SOC group.

Sairenchi et $a l^{20}$ reported that during a one year follow-up of depression in Japanese workers, the odds ratio of the high SOC group for future depression, compared to the low SOC group, was 0.18 (95\% CI, 0.04-0.79), indicating that the high SOC group had a lower risk of future depression than the low SOC group. In our study, the reference was the high SOC group, hence when we re-calculated the odds ratio in Sairenchi's study using their high SOC group as the reference, the odds ratio of their low SOC group for future depression was 5.56, which is somewhat higher than that of our present study, however our results are still comparable to Sairenchi's study. In another previous study investigating the association between SOC scores and mental health among Japanese workers, the PSRs (Psychological Stress Response Scale) was used as a tool for evaluating mental health; to screen positive for PSRs means that the latent stress response is relatively high. In this study, the odds ratio for screening positive for PSRs was 6.35 in the low SOC group and 1.48 in the middle group (the reference being the high SOC group) ${ }^{28}$ These results, while somewhat higher than our odds ratios, still have a similarity to our present results.

In univariate analysis for new-onset depressive symptoms, the mean weekly working time of residents with depressive symptoms was higher than those without 
depressive symptoms (83.1 hours vs. 77.5 hours). The mean weekly working time also had a significant independent association with new-onset depressive symptoms, and its odds ratio was 1.04. Since a resident working more than 80 hours a week has a tendency for an increased risk of impaired mental health ${ }^{29}$ or medical error, ${ }^{30}$ the working hours of residents are restricted to less than 80 hours in the US ${ }^{11}$ and Europe. ${ }^{22}$ In the present study, $45.7 \%$ of residents were working more than 80 hours per week, so it seems important to regulate the working time of residents to care for their mental health.

The job stress model of the National Institute of Occupational Safety and Health (NIOSH), ${ }^{31}$ which is one of the most widely used occupational stress models, ${ }^{22}$ may help explain a possible mechanism for how the SOC score may predict a group at high risk of depressive symptoms. This NIOSH model suggests that exposure to stressful working conditions (e.g. long working hours, complicated inter-personal relationships) can have a direct influence on worker safety and health, and can generate a stress reaction such as depression or burn-out. ${ }^{32}$ But individual factors (e.g. job position, relaxed and positive outlook) and other situational factors (e.g. support from colleagues or supervisors, work-home balance) can intervene to strengthen or weaken this influence. According to the NIOSH job stress model, when residents face the same stressful working conditions, but have different levels of stress-coping ability, which is thought to be an individual factor, the perceived level of influence on their mental state would be different. Therefore, since the SOC scale can measure this stress-coping ability, the SOC score may well predict future depression, as the NIOSH model shows that one of the important reactions to stress is depression.

Currently, general preventive interventions, such as restricting working time ${ }^{11}$ and assistance services for residents, ${ }^{3}$ are being used to provide mental health care for 
residents. However, even with these interventions, a significant number of residents still have depressive symptoms. Since the SOC scale may be able to predict the group at high risk of future depressive symptoms, this high risk group could be carefully monitored and early signs of depressive symptoms detected, and appropriate care provided before the symptoms worsen. ${ }^{6}$ The SOC scale is an easy-to-use tool and needs just a few minutes to administer, making it feasible for many educational hospitals. Furthermore, because the SOC score could be evaluated before the start of clinical training, there would be enough time to prepare support for the high risk group, such as frequent short-term counselling sessions, ${ }^{6}$ and sharing the issue with directors and attending physicians. ${ }^{3}$

Maintaining the stress-coping ability of residents is also an important issue. Antonovsky stated that a SOC is basically developed by around age 30, and can be maintained by positive, supportive, and meaningful life events. ${ }^{33}$ Thus, in the case of residents, positive work situations or events, such as gratitude from patients, support from colleagues and job satisfaction, might influence the stress-coping ability of residents and would be helpful in maintaining their SOC.

While the SOC scale appears to be a useful tool, we should be careful in which situations it is used. In fact, while the odds ratio of the low SOC group for new-onset depressive symptoms was threefold higher than the high SOC group, 22.3\% of residents had new-onset depressive symptoms in middle SOC group, $11.3 \%$ of residents had new-onset depressive symptoms in high SOC groups. Therefore, a general and comprehensive strategy for preventive interventions for depression is obviously needed for all residents, as depression can be influenced by many related factors.

This study has some limitations. First, we could only distribute questionnaires to residents who belonged to the limited number of hospitals that agreed to participate in this 
study. In addition, we only included the residents who responded to both the first and follow-up surveys. It is possible that the residents who responded to our questionnaire were interested in issues such as working conditions, mental health, or depression. In contrast, it is possible that severely depressed residents could not respond to this survey. Therefore, we may have over-estimated or under-estimated the number of residents with new-onset depressive symptoms.

Second, only 251 of 853 hospitals (29.4\%) participated in this study, which is a relatively small number. However, the hospitals that participated in the study were geographically spread across Japan. Moreover, of the residents who participated in this study, $39.7 \%$ belonged to university hospitals and $60.3 \%$ belonged to other educational hospitals, which might not represent the total distribution of residents in Japan (45.2\% in university hospitals, 54.8\% in other educational hospitals). ${ }^{34}$ However there was no significant difference in new-onset depressive symptoms between residents belonging to university hospitals and other educational hospitals, so the results should not be influenced substantially.

Third, the follow-up survey was conducted only once, three months after the baseline survey; hence the present study could not clarify the prevalence of depression further in the future. A previous study, however, suggested that the prevalence of depression is highest after three months of clinical training among first year residents, ${ }^{35}$ thus our follow-up survey was timed to find out the prevalence of new-onset depressive symptoms three months after the start of clinical training.

Finally, we used a self-administered questionnaire and defined residents who had a CES-D score of 16 or more as screening positive for depressive symptoms. Therefore, it should be noted that this may not always match a diagnosis of depression, or detect 
residents who actually have depressive symptoms associated with depression. However in the present study, the prevalence of depressive symptoms was $15.6 \%$ at the baseline survey, which was similar to the $15.2 \%$ prevalence of depression in a study in which the

CES-D score was translated into Japanese. ${ }^{24}$ Moreover, including residents with depressive symptoms at baseline, 30.3\% of residents had depressive symptoms at the follow-up survey. This is comparable to previous studies using the CES-D as a screening tool for depressive symptoms among medical residents which reported the prevalence of such symptoms as $29.2 \%$ to $34.2 \%$. $^{1,36}$ This confirms some amount of value and accuracy in the use of self-administered questionnaires.

\section{CONCLUSIONS}

The SOC scale, evaluated before the start of clinical training, could help identify medical residents at high risk of depressive symptoms, so they can be provided with effective support. It is of value to both residents and medical educators. 
Contributors: Contributors: MI contributed to data analysis and interpretation, and the drafting of the article. ES contributed to the study concept and design, data acquisition and analysis, interpretation, and the revision of the manuscript. OR contributed to data acquisition and analysis, interpretation and the revision of the manuscript. MS contributed to data acquisition and analysis, interpretation and the revision of the manuscript. TM contributed to data analysis and interpretation, and the revision of the manuscript. TM contributed to the study concept and design, the revision of the manuscript, and the final approval of the version to be published.

We all have agreement to be accountable for all aspects of our work in ensuring that questions related to the accuracy or integrity of any part of our work are appropriately investigated and resolved. And all authors approved the final manuscript for publication.

Acknowledgements: The authors wish to acknowledge all medical residents and training program coordinators for their generous cooperation.

Funding: This work was supported JSPS KAKENHI Grant Number 22590452.

Conflicts of interest: None.

Ethical approval: The study was approved by the Ethics Committee of the Faculty of Medicine of the University of Tsukuba. 


\section{References}

1 Sakata Y, Wada K, Tsutsumi A, et al. Effort-reward imbalance and depression in Japanese medical residents. J Occup Health 2008;50:498-504.

2 Tyssen R, Vaglum P, Gronvold NT, Ekeberg O. Factors in medical school that predict postgraduate mental health problems in need of treatment. A nationwide and longitudinal study. Med Educ 2001;35:110-20.

3 Levey RE. Sources of stress for residents and recommendations for programs to assist them. Acad Med 2001;76:142-50.

4 Michels PJ, Probst JC, Godenick MT, Palesch Y. Anxiety and anger among family practice residents: a South Carolina family practice research consortium study. Acad Med 2003;78:69-79.

5 Tyssen R, Vaglum P. Mental health problems among young doctors: an updated review of prospective studies. Harv Rev Psychiatry 2002;10:154-65.

6 Brooks SK, Gerada C, Chalder T. Review of literature on the mental health of doctors: are specialist services needed? J Ment Health.2011;20:146-56.

7 Goebert D, Thompson D, Takeshita J, et al. Depressive symptoms in medical students and residents: a multischool study. Acad Med 2009;84:236-41.

8 Hochberg MS, Berman RS, Kalet AL, Zabar SR, Gillespie C, Pachter HL. The stress of residency: recognizing the signs of depression and suicide in you and your fellow residents. Am J Surg 2013;205:141-6.

$9 \quad$ Fahrenkopf AM, Sectish TC, Barger LK, et al. Rates of medication errors among depressed and burnt out residents: prospective cohort study. BMJ 2008;336:488-91. 
10 Wu AW. Medical error: the second victim. The doctor who makes the mistake needs help too. BMJ 2000;320:726-7.

11 Tyssen R, Vaglum P, Gronvold NT, Ekeberg O. The impact of job stress and working conditions on mental health problems among junior house officers. A nationwide Norwegian prospective cohort study. Med Educ 2000;34:374-84.

12 Stoen Grotmol K, Gude T, Moum T, Vaglum P, Tyssen R. Risk factors at medical school for later severe depression: a 15-year longitudinal, nationwide study (NORDOC). J Affect Disord 2013;146:106-11.

13 Katz ED, Sharp L, Ferguson E. Depression among emergency medicine residents over an academic year. Acad Emerg Med 2006;13:284-7.

14 Sen S, Kranzler HR, Krystal JH, et al. A prospective cohort study investigating factors associated with depression during medical internship. Arch Gen Psychiatry 2010;67:557-65.

15 Murphy JM, Laird NM, Monson RR, Sobol AM, Leighton AH. A 40-year perspective on the prevalence of depression: the Stirling County Study. Arch Gen Psychiatry 2000;57:209-15.

16 Maeno T, Nakamura A, Maeno T, et al. Resident stress in the new postgraduate clinical training system.[in Japanese.] Igaku Kyoiku 2008;39:175-82.

17 Vetto JT, Robbins D. Impact of the recent reduction in working hours (the 80 hour work week) on surgical resident cancer education. J Cancer Educ 2005;20:23-7.

18 Baldwin PJ, Dodd M, Wrate RW. Young doctors' health--I. How do working conditions affect attitudes, health and performance? Soc Sci Med 1997;45:35-40.

19 Brewin CR, Firth-Cozens J. Dependency and self-criticism as predictors of depression in young doctors. J Occup Health Psychol 1997;2:242-6. 
20 Sairenchi T, Haruyama Y, Ishikawa Y, Wada K, Kimura K, Muto T. Sense of coherence as a predictor of onset of depression among Japanese workers: a cohort study. BMC Public Health 2011;11:205.

21 Konttinen H, Haukkala A, Uutela A. Comparing sense of coherence, depressive symptoms and anxiety, and their relationships with health in a population-based study. Soc Sci Med 2008;66:2401-12.

22 Haoka T, Sasahara S, Tomotsune Y, Yoshino S, Maeno T, Matsuzaki I. The effect of stress-related factors on mental health status among resident doctors in Japan. Med Educ 2010;44:826-34.

23 Radloff LS. The CES-D scale: a self-report depression scale for research in the general population. Appl Psychol Meas 1977;1:385-401.

24 Shima S, Shikano T, Kitamura T, Asai M. New self-rating scales for depression.[in Japanese.] Seisin Igaku 1985;27:717-23.

25 Togari T, Yamazaki Y. Examination of the reliability and factor validity of 13-item five-point version Sense of Coherence Scale.[in Japanese.] Japanese Journal of Health and Human Ecology 2005;71:168-82.

26 Tatsukawa H, Sasahara S, Yoshino S, et al. Influences of the stress coping ability of supervisors on the stress situation of their subordinate. Tairyoku Eiyo Menekigaku Zasshi 2005;15:82-7.

27 Taniguchi K, Sasahara S, Maeno T, et al. Working environment, job stress factor and mental health among Japanese medical residents and supervisors. Tairyoku Eiyo Menekigaku Zasshi 2007;17:223-9.

28 Umeda T, Kobayasi N, et al. Research for influence of sense of coherence on mental health among worker: A large-scale epidemiologic survey of workers 
during one year.[in Japanese] Tairyoku Eiyo Menekigaku Zasshi 2010;20:183-5.

29 Gopal R, Glasheen JJ, Miyoshi TJ, Prochazka AV. Burnout and internal medicine resident work-hour restrictions. Arch Intern Med 2005;165:2595-600.

30 Jagsi R, Weinstein DF, Shapiro J, Kitch BT, Dorer D, Weissman JS. The Accreditation Council for Graduate Medical Education's limits on residents' work hours and patient safety. A study of resident experiences and perceptions before and after hours reductions. Arch Intern Med 2008;168:493-500.

$31 \quad$ NIOSH. STRESS...At Work. DHHS (NIOSH) Publication No. 99-101; 1999.

32 Hurrell JJ Jr, McLaney MA. Exposure to job stress--a new psychometric instrument. Scand J Work Environ Health 1988;14 (Suppl 1):27-8.

33 Antnovsky A. Unraveling the mystery of health: how people manage stress and stay well .(Japanese version) Tokyo: Ushindo 2001.

34 The data of clinical training of Japan. http://www.jrmp.jp/ [accessed 21 March 2014.].

35 Stress and impairment during residency training: strategies for reduction, identification, and management. Resident Services Committee, Association of Program Directors in Internal Medicine. Ann Intern Med 1988;109:154-61.

36 Becker JL, Milad MP, Klock SC. Burnout, depression, and career satisfaction: cross-sectional study of obstetrics and gynecology residents. Am J Obstet Gynecol 2006;195:1444-9. 
Table 1. Participant characteristics, mean SOC score, SOC category, and mean working time $(\mathrm{n}=1020)$

Age, mean $\pm S D$, y

$26.0 \pm 3.0$

Sex

Male, n (\%)

$668(65.5 \%)$

Female, n (\%)

$352(34.5 \%)$

SOC score

SOC score, mean \pm SD

$44.4 \pm 6.0$

Low, n (\%)

$148(14.5 \%)$

Middle, n (\%)

$712(69.8 \%)$

High, n (\%)

$160(15.7 \%)$

\section{Hospital type}

University hospital, n (\%)

$405(39.7 \%)$

Other educational hospital, n (\%)

$615(60.3 \%)$

Working time ${ }^{1}$

Mean \pm SD, hours/week

$78.8 \pm 13.6$

Residents working more than 80 hours/ week, n(\%)

$375(45.7 \%)$

${ }^{1} n=820$ as 200 residents returned the survey but did not provide an answer for working conditions.

Abbreviations: SOC, sense of coherence; SD, standard deviation. 
Table 2. Association of new-onset depressive symptoms with demographic factors, SOC score, baseline CES-D score, and mean working time

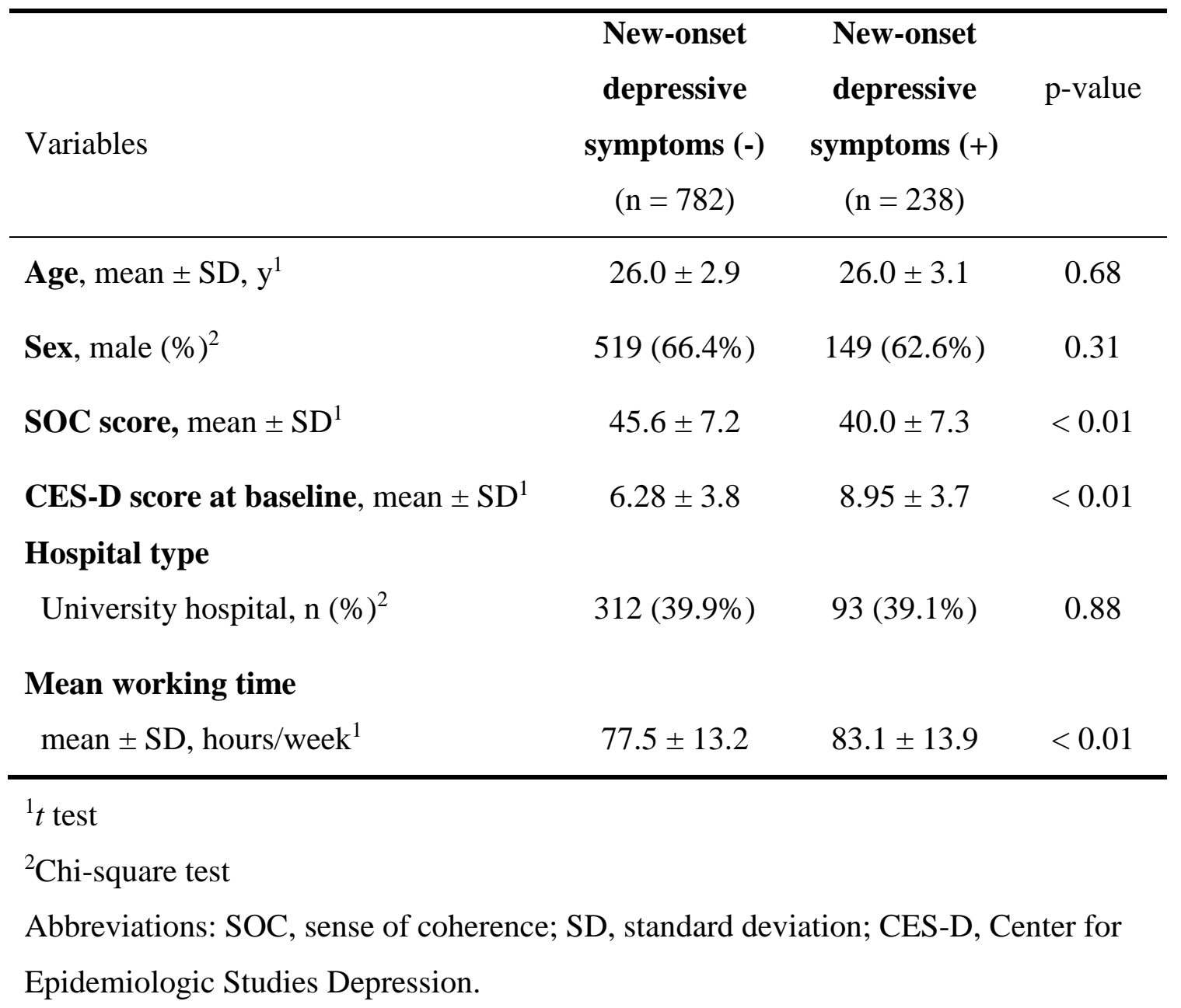


Figure 1. The relationship between the number of residents with new-onset depressive symptoms and the three SOC score groups

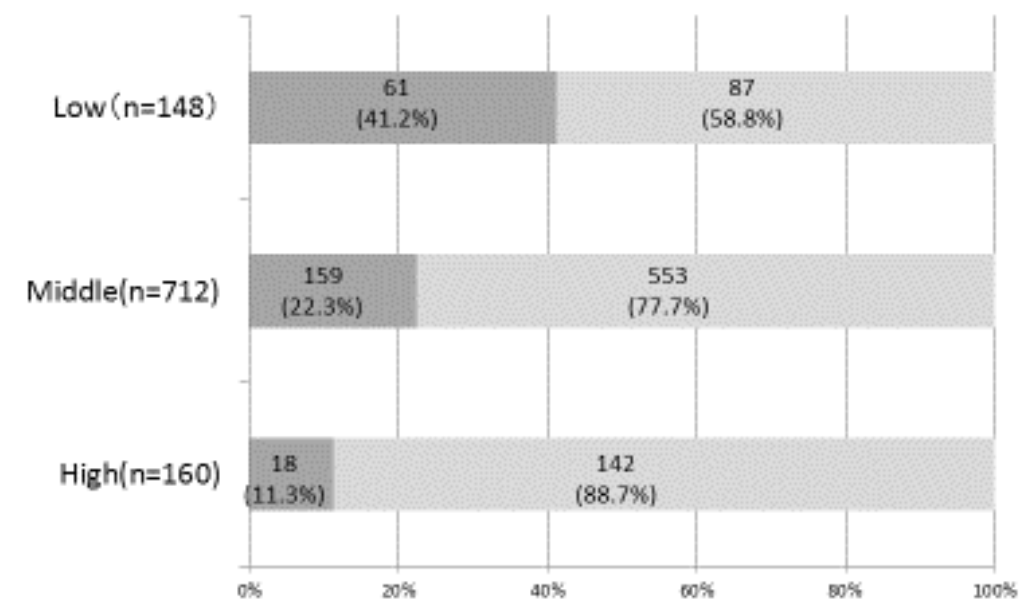

Wn-w-onset depressive symptoms(+) new-onset depressive symptoms(-)

Abbreviation: SOC, sense of coherence

Chi-square test $p<0.01$

Prevalences of new-onset depressive symptoms in residents $(n=1020)$ grouped according to baseline scores on the Sense of Coherence Scale. Chi-squared test, $\mathrm{p}<0.01$ 
Table 3. Association between new-onset depressive symptoms and the SOC score $(\mathbf{n}=\mathbf{8 2 0})$

\begin{tabular}{lccc}
\hline & Odds ratio & $95 \%$ CI & p-value \\
\hline SOC score groups & 3.11 & $1.48-6.53$ & $<0.01$ \\
$\quad$ Low & 1.72 & $0.92-3.24$ & 0.09 \\
$\quad$ Middle & 1.00 & & \\
$\quad$ High & 1.17 & $1.12-1.23$ & $<0.01$ \\
CES-D score at baseline & 1.05 & $0.99-1.12$ & 0.11 \\
Age & 1.30 & $0.90-1.89$ & 0.16 \\
Sex & & & \\
Hospital type & 0.87 & $0.61-1.25$ & 0.45 \\
Mean working time & 1.04 & $1.02-1.05$ & $<0.01$ \\
\hline
\end{tabular}

The results of a binomial logistic regression analysis where the dependent variable is new-onset depressive symptoms and the independent variables are the three SOC groups, CES-D score at baseline, age, sex, hospital type, and mean working time.

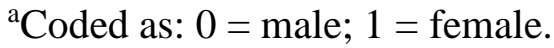

${ }^{\text {b}}$ Coded as: 0 = university hospital; 1 = other educational hospital.

Abbreviations: CI, confidence interval; SOC, sense of coherence; CES-D, Center for Epidemiologic Studies Depression. 